\title{
Leukoaraiosis Predicts a Poor 90-Day Outcome after Endovascular Stroke Therapy
}

\author{
J. Zhang, A.S. Puri, M.A. Khan, R.P. Goddeau Jr, and N. Henninger
}

\begin{abstract}
BACKGROUND AND PURPOSE: Leukoaraiosis is a common finding among patients with ischemic stroke and has been associated with poor stroke outcomes. Our aim was to ascertain whether the severity of pre-existing leukoaraiosis is associated with outcome in patients with acute ischemic stroke who are treated with endovascular stroke therapy.
\end{abstract}

MATERIALS AND METHODS: We retrospectively analyzed data from 129 consecutive, prospectively enrolled patients with stroke undergoing endovascular stroke therapy at a single tertiary care center between January 2006 and August 2013. Leukoaraiosis was assessed as supratentorial white matter hypoattenuation on admission head CT and graded as 0-2 (absent-to-moderate) versus 3-4 (severe) according to the van Swieten scale. We dichotomized the 90-day mRS into good (0-2 or return to baseline) versus poor (3-6) as the primary study outcome. Incremental multivariable logistic regression analyses were performed to identify independent predictors of a poor 90-day outcome.

RESULTS: In all multivariable models, severe leukoaraiosis was independently $(P<.05)$ associated with a poor outcome at 90 days (fully adjusted model: OR, 6.37; 95\% Cl, 1.83-12.18; $P=.004)$. The independent association between leukoaraiosis and a poor outcome remained when the analysis was restricted to patients who were alive at discharge $(n=87, P<.05)$. Last, among patients who were alive at discharge, those with severe leukoaraiosis had significantly less frequent improvement on the mRS from discharge to 90 days compared with patients with absent-to-moderate leukoaraiosis $(P=.034)$.

CONCLUSIONS: The severity of pre-existing leukoaraiosis is independently associated with 90-day functional outcome in patients with stroke who underwent endovascular stroke therapy. These results highlight the need to further explore leukoaraiosis as a promising surrogate marker for poor outcome after endovascular stroke therapy to improve risk assessment, patient selection, and early prognostic accuracy.

ABBREVIATION: EST $=$ endovascular stroke therapy

$\mathrm{T}$ hrombolysis with IV rtPA remains the only proved therapy for acute ischemic stroke. ${ }^{1}$ However, its efficacy is limited by several variables, including a narrow treatment time window and reduced efficacy with larger thrombus burden. ${ }^{1,2}$ In particular, proximal large intracranial artery occlusion portends a poor

Received March 27, 2014; accepted after revision May 12.

From the Departments of Neurology (J.Z., N.H., R.P.G.), Radiology (A.S.P.), Neurosurgery (A.S.P.), and Psychiatry (N.H.), University of Massachusetts Medical School, Worcester, Massachusetts; and Department of Neurology (M.A.K.), Warren Alpert Medical School of Brown University, Providence, Rhode Island.

Please address correspondence to Nils Henninger, MD, Departments of Neurology and Psychiatry, University of Massachusetts Medical School, 55 Lake Ave, North, Worcester, MA 01655; e-mail: nils.henninger@umassmed.edu

三 Indicates article with supplemental on-line tables.

Indicates article with supplemental on-line figures.

http://dx.doi.org/10.3174/ajnr.A4029 prognosis and poses a substantial therapeutic challenge. ${ }^{3}$ Endovascular approaches to recanalization may allow more frequent and rapid recanalization of the target vessel, which is critical for achieving better outcomes. ${ }^{2,3}$ However, despite the acceptable safety of endovascular approaches, definitive proof of superiority over IV thrombolysis in achieving good functional outcomes remains to be established. ${ }^{1,3}$ Understanding factors that influence outcome in patients undergoing endovascular stroke therapy (EST) is paramount to help improve selection of patients most likely to benefit from this therapy. ${ }^{2}$

While numerous studies have focused on procedural aspects, treatment window, and neuroimaging signatures of acute tissue injury, little is known regarding the potential contribution of preexisting white matter rarefaction (leukoaraiosis) on outcome. ${ }^{3-5}$ Yet, leukoaraiosis is frequently encountered in the elderly ${ }^{6,7}$ and is well-known to contribute to worse outcomes after acute ischemic 
stroke. ${ }^{7,8}$ Given the expected tripling of the number of persons aged 60 or older in developed nations by $2050^{9}$ and the increasing prevalence of stroke with advancing age, ${ }^{10,11}$ it is expected that an increasing number of patients with pre-existing leukoaraiosis will be treated with EST. ${ }^{12}$ Accordingly, it is important to understand the potential contribution of leukoaraiosis to functional outcome after EST.

To address this issue, we tested the hypothesis that the severity of pre-existing leukoaraiosis is associated with outcome as assessed by the 90 -day mRS in consecutive patients with acute ischemic stroke treated with EST.

\section{MATERIALS AND METHODS \\ Study Population}

This study was reviewed and approved by our institutional review board. We retrospectively analyzed 129 consecutive, prospectively collected patients with acute ischemic stroke who underwent EST at a single academic stroke center from January 2006 to August 2013. Of note, 42 (33\%) studied subjects have been included in prior investigations. ${ }^{8,13}$ We adhered to the STrengthening the Reporting of OBservational studies in Epidemiology guidelines (www.strobe-statement.org).

All patients underwent head CT and CTA at admission. Demographics, NIHSS scores, laboratory data, comorbidities, preadmission medications, and stroke etiology (by using the Trial of Org 10172 in Acute Stroke Treatment classification), ${ }^{8,13}$ after completion of diagnostic evaluation, were collected on all patients. mRS scores were assessed at the time of presentation (preadmission mRS), discharge, and at 90 days by a stroke-trained physician or stroke study nurse, certified in $\mathrm{mRS}^{8}{ }^{8}$ When the mRS was unavailable, the same observers reconstructed the score from the case description, according to the mRS criteria. ${ }^{8}$

\section{Neuroimaging Protocol}

All CT sequences were obtained on a 64-detector row scanner (Brilliance; Philips Healthcare, Best, the Netherlands). CT was performed in a nonhelical mode at $120 \mathrm{kV}$ (peak) and $200 \mathrm{~mA}$, with data reconstruction at $5-\mathrm{mm}$ axial sections. CTA was performed by using $64 \times 0.625 \mathrm{~mm}$ detector configuration with a pitch of 0.673 from the arch of the aorta to the vertex by using 120 $\mathrm{kV}(\mathrm{p}), 300 \mathrm{~mA}$, and 0.5 -second rotation time. Patients received 60-80 mL of iopamidol (Isovue 370; Bracco Diagnostics, Princeton, New Jersey) in the antecubital vein at a rate of $4 \mathrm{~mL} / \mathrm{s}$ through a power injector followed by $40 \mathrm{~mL}$ of saline. 3D orthogonal MIP images were created in 3 planes.

\section{Digital Subtraction Angiography}

All endovascular procedures were performed by senior neurointerventionalists by using a biplane angiography unit with 3D rotational angiography capability (Allura Xper FD20/20; Philips) with patients under conscious sedation or general anesthesia. Endovascular access was obtained by a standard transfemoral approach.

\section{Image Review and Analysis}

CT and CTA were reviewed independently by experienced readers blinded to both clinical data and any follow-up scans. Variable window width and center-level settings were used for optimal ischemic hypoattenuation detection with CT and CTA. ${ }^{8,13}$

Leukoaraiosis was retrospectively defined by 2 readers (N.H. and J.Z.) as supratentorial white matter hypoattenuation on admission noncontrast head CT according to the STandards for ReportIng Vascular changes on nEuroimaging criteria, ${ }^{14}$ and it was graded on a 5-point van Swieten scale as previously described in detail (On-line Fig 1). ${ }^{15}$ Disagreements in readings were resolved by consensus. In the present study, leukoaraiosis was separately assessed in each hemisphere, but only the score from the nonischemic hemisphere was considered after unblinding. Hemorrhagic transformation on follow-up imaging was determined according to the European Cooperative Acute Stroke Study definition. ${ }^{16}$

Angiography-based collateral flow was graded from 0 (no collaterals) to 4 (complete) before EST, ${ }^{17}$ and final recanalization was assessed according to the TICI classification as previously defined. ${ }^{17}$ The time to recanalization was defined as the time from symptom onset (or last time known well) to the final attempt at recanalization.

To avoid classification bias and to minimize interrater variability, ${ }^{8}$ we also dichotomized the degree of leukoaraiosis (van Swieten scale $0-2$ [absent-to-moderate] versus 3-4 [severe]), the presence of collaterals (grades $0-2$ [poor] versus 3-4 [good]), recanalization (TICI $0-2 \mathrm{a}$ [poor] versus $2 \mathrm{~b}-3$ [good]), and the presence versus absence of parenchymal hemorrhage (parenchymal hemorrhage 1 and parenchymal hemorrhage 2) for statistical purposes.

Last, to assess the potential association among leukoaraiosis, infarct volume, and outcome, manual lesion assessment ${ }^{13,15}$ was conducted in patients who had follow-up imaging within 7 days of stroke onset (MR imaging: $n=43$; CT: $n=25$ ), allowing reliable measurements.

\section{Statistics}

Weighted $\kappa$ statistics were used to determine the degree of interrater agreement in the van Swieten scale assessment in all 129 included patients and to determine $\kappa$ values as previously defined. ${ }^{13}$ In addition, intrarater agreement was assessed in 65 randomly chosen patients who were graded 5 months apart by an experienced reader (N.H.).

Unless otherwise stated, continuous variables are reported as mean $\pm \mathrm{SD}$ or as median (interquartile range). Categoric variables are reported as proportions. Between-group comparisons for continuous variables were made with the unpaired $t$ and Mann-Whitney $U$ tests, as appropriate. Categoric variables were compared by using the $\chi^{2}$ or Fisher exact test as appropriate.

Primary Analysis. We defined the 90-day mRS dichotomized into good (0-2 or return to baseline) versus poor (3-6) as the primary study outcome. To test the hypothesis that the degree of leukoaraiosis was independently associated with outcome, we created several incremental multivariable logistic regression models as follows: first unadjusted (model 1); then adjusted for admission variables: age, NIHSS, atrial fibrillation, creatinine level, lowdensity lipoprotein cholesterol, random blood sugar (model 2); then additionally adjusted for endovascular variables: poor recanalization, time to recanalization, poor collateral grade (model 3); 


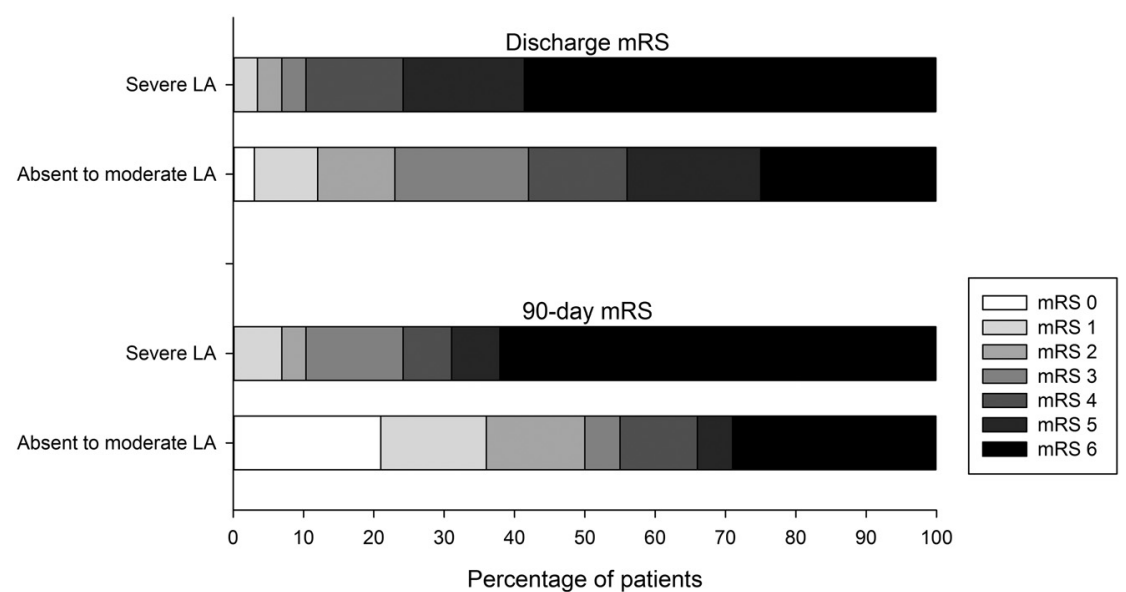

FIG 1. Functional outcome at discharge and at 3 months according to leukoaraiosis (LA) severity (unadjusted). Scores of $\leq 2$ or return to baseline on the mRS are considered a good outcome (darker shades indicate worse outcomes).

and finally additionally adjusted for the presence of parenchymal hemorrhage (model 4). We then repeated all analyses, entering leukoaraiosis severity, collateral grade, and degree of recanalization as ordinal variables.

Secondary Analyses. We performed preplanned sensitivity analyses for predictors of a good (0-2 or return to baseline) versus poor (3-6) mRS at 90 days in patients who were alive by the time of discharge $(n=87)$. Furthermore, we compared the frequency of any improvement (defined as an improvement by $\geq 1$ point on the mRS between discharge and 90 days) in patients with absentto-moderate versus severe leukoaraiosis who were alive at the time of discharge. Last, we conducted multivariable linear regression analysis with backward elimination of factors independently associated with the final infarct volume. We performed multivariable logistic regression analyses (by using bootstrapping for more stable results, given the limited number) to assess whether leukoaraiosis and infarct volume were independently associated with a poor 90-day outcome.

Collinearity diagnostics were performed for all multivariable logistic and linear regression models. Associations are presented as ORs with corresponding 95\% confidence intervals. The HosmerLemeshow goodness-of-fit statistic was used to assess all models for final fit. Two-sided significance tests were used throughout, and a 2 -sided $P<.05$ was considered statistically significant. All statistical analyses were performed by using SPSS Statistics 20.0.0 (IBM, Armonk, New York).

\section{RESULTS}

During the study period, 3057 patients were admitted with a diagnosis of acute ischemic stroke. Of these, 142 patients were treated with EST. Thirteen patients were lost to follow-up. Data were complete for all included patients except for collateral grade, which could not be assessed in 8 patients due to proximal internal carotid artery occlusion and absent contralateral injection before the intervention.

Free marginal $\kappa$ values for interobserver agreement indicated moderate and almost perfect agreement for graded $(\kappa=$ $0.50)$ and dichotomized leukoaraiosis severity $(\kappa=0.91)$, respectively. ${ }^{13}$ Intraobserver agreement was substantial and almost perfect for graded $(\kappa=0.78)$ and dichotomized leukoaraiosis severity $(\kappa=0.89)$, respectively.

\section{Factors Associated with} Leukoaraiosis Severity (Unadjusted) Baseline characteristics of the 129 included patients stratified by leukoaraiosis severity and outcome are summarized in On-line Table 1. Severe leukoaraiosis was associated with greater in-hospital mortality $(P=.001)$, worse $\mathrm{mRS}$ scores at discharge $(P=.003)$, and worse 90-day outcome $(P=.001$, Fig 1$)$. Furthermore, patients with severe leukoaraiosis were more likely to have experienced a prior stroke or TIA $(P=.002)$, atrial fibrillation $(P=.001)$, coronary artery disease $(P=$ $.030)$, or peripheral vascular disease $(P=$ $.009)$, were older $(P<.001)$, less frequently underwent mechanical thrombectomy, and had more frequent cardioembolic strokes $(P=.005)$ and strokes of other or undetermined pathogenesis $(P=.039)$.

There was a weak inverse relation between leukoaraiosis and collateral grade (ie, patients with worse leukoaraiosis had better collaterals; graded: $r=-.218, P=.016$; dichotomized: $r=$ $-.265, P=.003$ ). There was no association between the severity of leukoaraiosis and the degree of recanalization (graded: $r=$ $0.083, P=.351$; dichotomized: $r=0.029, P=.743$ ).

\section{Factors Associated with a Poor 90-Day Outcome (Unadjusted)}

Poor 90-day outcome (On-line Table 2) was associated with older age $(P<.001)$ and worse leukoaraiosis (graded: $P=.005$; dichotomized: $P<.001)$ and higher admission NIHSS scores $(P<.001)$, glucose levels $(P=.032)$, creatinine levels $(P=$ $.02)$, and low-density lipoprotein levels $(P=.031)$, good recanalization $(P=.023)$, the presence of atrial fibrillation $(P=$ .014 ), and the presence of parenchymal hemorrhage ${ }^{16}$ (parenchymal hemorrhage 1 and parenchymal hemorrhage 2, $P=$ $.001)$. The association between leukoaraiosis severity and 90day outcome was similar across the endovascular modalities used (On-line Fig 2).

\section{Primary Analysis: Multivariable Analysis of Factors Associated with the 90-Day Outcome}

The association between severe leukoaraiosis and poor 90-day outcome remained after adjusting for pertinent covariates (Table). The association of leukoaraiosis with outcome remained when leukoaraiosis severity, degree of recanalization, and collateral grade were entered as ordinal variables in all models $(P<.01$, not shown).

\section{Secondary Analyses}

Association between Leukoaraiosis and Outcome among Patients Alive at Discharge. By the time of discharge, 33\% $(n=42)$ of patients had died (Fig 1, all because of "withdrawal of care"). To 
Multivariable logistic regression analysis of factors independently associated with a poor 90 -day outcome

\begin{tabular}{|c|c|c|c|c|c|c|c|c|}
\hline Independent Variable & Model $1^{a}$ & $P$ Value & Model $2^{b}$ & $P$ Value & Model $3^{c}$ & $P$ Value & Model $4^{d}$ & $P$ Value \\
\hline Severe leukoaraiosis ${ }^{\mathrm{e}}$ & $6.50(2.11-20.06)$ & $<.001$ & $4.40(1.22-15.74)$ & .023 & $6.03(1.80-22.11)$ & .004 & $6.37(1.83-12.18)$ & .004 \\
\hline Admission NIHSS score & - & - & 1.08 (1.00-1.16) & .027 & 1.09 (1.01-1.17) & .023 & 1.08 (1.00-1.16) & .041 \\
\hline Age (yr) & - & - & $1.03(0.99-1.07)$ & .065 & $1.03(1.00-1.07)$ & .028 & $1.03(0.99-1.06)$ & .005 \\
\hline LDL & - & - & $0.98(0.97-1.00)$ & .057 & $0.98(0.97-1.00)$ & .014 & $0.99(0.97-1.00)$ & .064 \\
\hline Creatinine level & - & - & - & - & $3.21(0.78-13.19)$ & .105 & - & - \\
\hline Poor recanalization & - & - & - & - & $3.72(1.10-12.53)$ & .034 & $2.94(0.84-10.21)$ & .089 \\
\hline Parenchymal hemorrhage & - & - & - & - & - & - & $3.23(1.01-10.37)$ & .049 \\
\hline
\end{tabular}

Note:- - indicates not applicable; LDL, low-density lipoprotein. Factors that were not included in the final model iteration are omitted from the table. Data are odds ratios with $95 \%$ confidence intervals.

${ }^{\text {a }}$ Model 1, unadjusted.

${ }^{\mathrm{b}}$ Model 2, adjusted for age, admission NIHSS score, atrial fibrillation, creatinine level, LDL, and random blood sugar (Hosmer-Lemeshow statistics: $\chi^{2}=6.047, P=.642$ )

${ }^{c}$ Model 3, additionally adjusted for collateral grade, degree of recanalization, and time to recanalization (Hosmer-Lemeshow statistics: $\left.\chi^{2}=9.856, P=.275\right)$.

${ }^{\mathrm{d}}$ Model 4, adjusted for model 3 plus the presence of parenchymal hemorrhagic transformation (Hosmer-Lemeshow statistics: $\chi^{2}=3.418, P=.905$ ).

e The association with outcome remained when leukoaraiosis, degree of recanalization, and collateral grade were entered as ordinal variables $(P<.01)$.

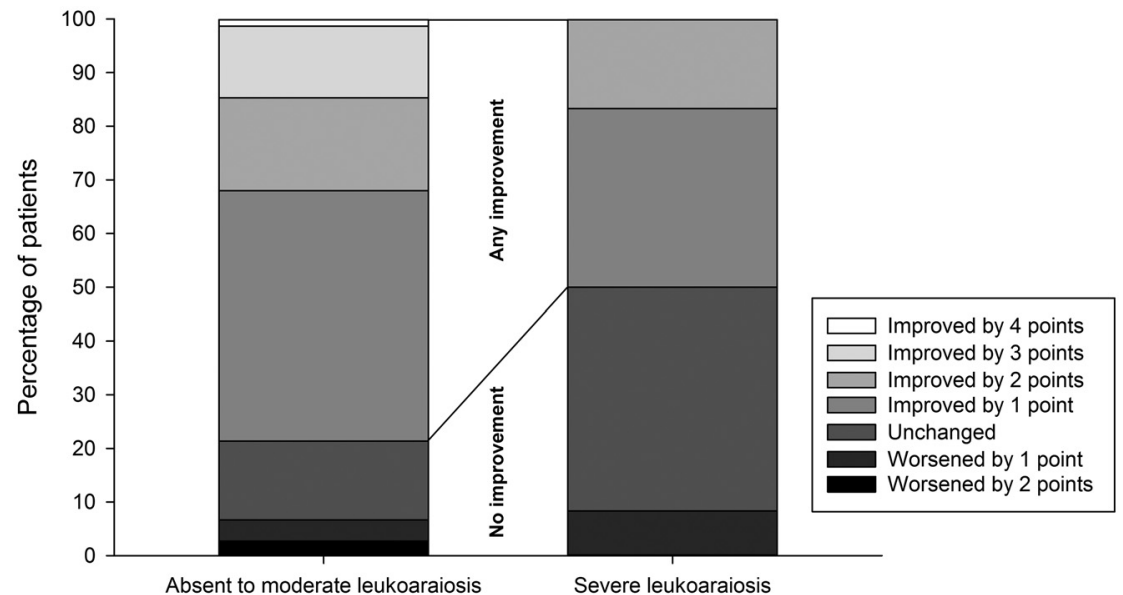

FIG 2. Functional improvement of patients who were alive at the time of discharge $(n=87)$ according to leukoaraiosis severity (unadjusted). Compared with patients with absent-to-moderate leukoaraiosis, patients with severe leukoaraiosis are significantly less likely to have any improvement on the mRS from discharge to 90 days $(50 \%$ versus $79 \%$ of surviving patients $[21 \%$ versus $59 \%$ of all patients, respectively]; $P=.034$; darker shades indicate less improvement).
Patients with a poor outcome $(n=29)$ had significantly larger infarcts compared with patients with a good outcome $(131 \pm 111 \mathrm{~mL}$ versus $20 \pm 16$ $\mathrm{mL}, P<.001)$. Multivariable linear regression analysis (including age, admission NIHSS, leukoaraiosis, time to recanalization, degree of recanalization, collateral grade, random blood sugar, atrial fibrillation, use of antihypertensive medications, and glycated hemoglobin) indicated that leukoaraiosis $(P<.001)$, degree of recanalization $(P<.001)$, and age $(P=.036)$ were independently associated with the final infarct volume (not shown). Last, multivariable logistic regression (including age, leukoaraiosis, infarct volume, and admission NIHSS score) indicated that severe leukoaraiosis $(P=.035)$, infarct gain a better understanding of whether leukoaraiosis can serve as a useful independent prognostic factor for outcome in patients who did not have early withdrawal of care (which may be related to factors independent of measured patient characteristics), we then repeated the logistic regression analyses in patients who were alive by discharge $(n=87)$. These analyses yielded similar results, showing an independent association between severe leukoaraiosis and poor outcome (unadjusted $P=.001$; adjusted $P<.05$, not shown).

Association between Leukoaraiosis and Recovery among Patients Alive at Discharge. Among patients who were alive at discharge, those with severe leukoaraiosis had significantly less frequent improvement on the mRS from discharge to 90 days compared with patients with absent-to-moderate leukoaraiosis (OR, 3.69; 95\% CI, 1.05-12.99; $P=.034$; Fig 2 ). Given the limited number of subjects in the outcome category, multivariable analysis to adjust for covariates was not attempted.

Association among Infarct Volume, Leukoaraiosis, and Outcome. Among patients with follow-up imaging $(n=68)$, those with severe leukoaraiosis $(n=11)$ had significantly larger infarcts compared with patients with absent-tomoderateleukoaraiosis $(152 \pm 145 \mathrm{~mL}$ versus $51 \pm 68 \mathrm{~mL}, P=.044)$. volume $(P=.006)$, and age $(P=.004)$ were independently associated with a poor outcome (not shown).

\section{DISCUSSION}

We demonstrate that pre-existing leukoaraiosis severity is associated with a poor 90-day outcome after EST, independent of established predictors of stroke outcome.

This finding is important because translating favorable angiographic results of endovascular treatment to correspondingly robust clinical outcomes is needed. Because endovascular approaches to acute ischemic stroke treatment are increasingly offered to older patients, despite a higher risk of poor outcome compared with younger patients, ${ }^{12}$ appropriate patient selection beyond procedural aspects represents an important goal in this population.

Imaging biomarkers are of particular interest, given that neuroimaging is a prerequisite to patient selection for acute therapy. Leukoaraiosis may represent a viable biomarker because it can be determined by standard neuroimaging and has been consistently shown to predict a higher risk for poor poststroke outcome..$^{5-8,15,18}$

How leukoaraiosis contributes to worse poststroke outcomes 
is incompletely understood. For example, leukoaraiosis has been associated with a greater risk for parenchymal hemorrhage after EST, which is a predictor of poor poststroke outcome. ${ }^{4}$ However, in the current study, we did not note a significant association between leukoaraiosis and parenchymal hemorrhage risk, possibly because we included patients treated with newer generation devices, which may have a lower risk for hemorrhagic transformation ${ }^{19}$ and our study was likely underpowered to detect a significant association. Although our results are consistent with a retrospective analysis of patients treated with EST, ${ }^{5}$ further research is required to elucidate the potential association between leukoaraiosis and the risk for hemorrhagic transformation in this patient population.

An alternate explanation for poor outcomes in patients with leukoaraiosis may be chronic cerebral hypoperfusion that results in reduced cerebral ischemic resilience and thus greater final lesion volumes, ${ }^{20}$ which are a known outcome predictor after EST. ${ }^{21}$ Indeed, we and others have previously shown that leukoaraiosis severity is associated with a larger ischemic core, mismatch lost, and final infarct volume, supporting this hypothesis. ${ }^{13,15,22}$ Indeed, subgroup analyses in patients with available follow-up imaging indicated that worse leukoaraiosis independently predicted larger infarct volumes. However, because infarct volumes could not be measured in all patients, additional studies are required to confirm the association among leukoaraiosis, infarct volume, and outcome. Furthermore, because the impact of leukoaraiosis on poststroke outcome was only partially explained by larger infarct extent in this and prior studies, ${ }^{18}$ it appears likely that the loss of compensatory network integrity results in decreased cerebral plasticity. For example, decreased integrity of fiber tracts within the unaffected hemisphere and purported compensatory pathways (such as the corticorubrospinal system) have been shown to impair poststroke recovery. ${ }^{23,24}$ Accordingly, global white matter changes such as those caused by leukoaraiosis are expected to adversely affect the potential for poststroke recovery. However, although several studies have linked leukoaraiosis to poor functional outcomes, there is a paucity of data regarding the degree of poststroke recovery, particularly in patients with large-artery occlusion. ${ }^{25-27}$

In this respect, our observation of significantly decreased longterm recovery in patients having undergone EST with severe leukoaraiosis is a novel and important finding, supporting the hypothesis that leukoaraiosis disrupts cerebral connectivity and plasticity. Considering that most patients undergoing EST have early functional deficits, ${ }^{3}$ it is critical to understand factors that modulate recovery. Our findings highlight the clinical importance of investigating leukoaraiosis in patients with stroke to better understand its predictive value for functional recovery after stroke.

The strengths of the present study relate to the relatively large sample size, a well-defined patient population, investigation of variables that have been associated with poststroke outcome, and blinded imaging assessment. Our study has limitations related to its retrospective design and should be considered hypothesisgenerating. EST was not standardized across patients, and most patients were treated with multiple modalities with or without rtPA. For this reason, it was not feasible to adjust our analysis to account for the various treatment approaches or perform subgroup analyses. Nevertheless, exploratory analysis indicated a similar association across treatment modalities. This finding is consistent with the presumed pathophysiology and described association with poor poststroke outcomes in numerous studies across heterogeneous patient populations, allowing better generalization of our results. ${ }^{4,8,18}$ Misclassification of leukoaraiosis severity is possible given the CT-based grading system. However, although MR imaging has a higher sensitivity and specificity to research leukoaraiosis compared with $\mathrm{CT},{ }^{14}$ the latter is more broadly available and routinely used in the hyperacute setting to evaluate patients with stroke. Furthermore, as noted, the classification used in our study is easy to perform, had a high inter- and intraobserver agreement, and does not require extensive postprocessing. ${ }^{8,13}$ Last, although our data show a strong association between leukoaraiosis and outcome, a causal relationship remains to be fully established.

\section{CONCLUSIONS}

In summary, with a projected increase in the occurrence of strokes, especially among the elderly, ${ }^{9-11}$ clinicians will be increasingly ${ }^{12}$ faced with the issue of appropriately selecting elderly patients with severe strokes for EST. Our data provide preliminary evidence that patients with severe pre-existing leukoaraiosis are at high risk for a poor functional outcome after EST. Given absent association with parenchymal hemorrhage and favorable outcome in a small subset of patients, the presence of severe leukoaraiosis currently does not justify exclusion from EST. Nevertheless, these results highlight the need to further explore leukoaraiosis as a promising surrogate marker for poor outcome after EST to improve risk assessment and aid in discussing expectations of different outcome types with family members. Further study is required to confirm our findings and investigate underlying mechanisms.

Disclosures: Muhib A. Khan-UNRELATED: Payment for Development of Educational Presentations: Cyberrounds, for the development of a Continuing Medical Education on atrial fibrillation and newer oral anticoagulants.

\section{REFERENCES}

1. Jauch EC, Saver JL, Adams HP Jr, et al. Guidelines for the early management of patients with acute ischemic stroke: a guideline for healthcare professionals from the American Heart Association/ American Stroke Association. Stroke 2013;44:870-947

2. Nogueira RG, Gupta R, Davalos A. IMS-III and SYNTHESIS expansion trials of endovascular therapy in acute ischemic stroke: how can we improve? Stroke 2013;44:3272-74

3. Broderick JP, Palesch YY, Demchuk AM, et al. Endovascular therapy after intravenous t-PA versus t-PA alone for stroke. $N$ Engl J Med 2013;368:893-903

4. Shi ZS, Loh Y, Liebeskind DS, et al. Leukoaraiosis predicts parenchymal hematoma after mechanical thrombectomy in acute ischemic stroke. Stroke 2012;43:1806-11

5. Jung S, Mono ML, Findling $\mathrm{O}$, et al. White matter lesions and intraarterial thrombolysis. J Neurol 2012;259:1331-36

6. Simoni M, Li L, Paul NL, et al. Age- and sex-specific rates of leukoaraiosis in TIA and stroke patients: population-based study. Neurology 2012;79:1215-22

7. Pantoni L. Cerebral small vessel disease: from pathogenesis and clinical characteristics to therapeutic challenges. Lancet Neurol 2010;9:689-701 
8. Henninger N, Lin E, Baker SP, et al. Leukoaraiosis predicts poor 90-day outcome after acute large cerebral artery occlusion. Cerebrovasc Dis 2012;33:525-31

9. United Nations Department of Economic and Social Affairs, Population Division. World Population Prospects. The 2006 Revision, Highlights, Working Paper No. ESA/P/WP. 202. Published 2007. http:// www.un.org/esa/population/publications/wpp2006/WPP2006_ Highlights_rev.pdf. Accessed May 12, 2014

10. Lozano R, Naghavi M, Foreman K, et al. Global and regional mortality from 235 causes of death for 20 age groups in 1990 and 2010: a systematic analysis for the Global Burden of Disease Study 2010. Lancet 2012;380:2095-128

11. Murray CJ, Vos T, Lozano R, et al. Disability-adjusted life years (DALYs) for 291 diseases and injuries in 21 regions, 1990-2010: a systematic analysis for the Global Burden of Disease Study 2010. Lancet 2012;380:2197-223

12. Duffis EJ, He W, Prestigiacomo CJ, et al. Endovascular treatment for acute ischemic stroke in octogenarians compared with younger patients: a meta-analysis. Int J Stroke 2014;9:308-12

13. Henninger N, Lin E, Haussen DC, et al. Leukoaraiosis and sex predict the hyperacute ischemic core volume. Stroke 2013;44:61-67

14. Wardlaw JM, Smith EE, Biessels GJ, et al. Neuroimaging standards for research into small vessel disease and its contribution to ageing and neurodegeneration. Lancet Neurol 2013;12:822-38

15. Henninger N, Khan MA, Zhang J, et al. Leukoaraiosis predicts cortical infarct volume after distal middle cerebral artery occlusion. Stroke 2014;45:689-95

16. Hacke W, Kaste M, Fieschi C, et al. Intravenous thrombolysis with recombinant tissue plasminogen activator for acute hemispheric stroke: the European Cooperative Acute Stroke Study (ECASS). JAMA 1995;274:1017-25

17. Higashida RT, Furlan AJ, Roberts $\mathrm{H}$, et al. Trial design and reporting standards for intra-arterial cerebral thrombolysis for acute ischemic stroke. Stroke 2003;34:e109-37

18. Arsava EM, Rahman R, Rosand J, et al. Severity of leukoaraiosis correlates with clinical outcome after ischemic stroke. Neurology 2009;72:1403-10

19. Nogueira RG, Gupta R, Jovin TG, et al. Predictors and clinical relevance of hemorrhagic transformation after endovascular therapy for anterior circulation large vessel occlusion strokes: a multicenter retrospective analysis of 1122 patients. J Neurointerv Surg 2014 Jan 8. [Epub ahead of print]

20. O’Sullivan M, Lythgoe DJ, Pereira AC, et al. Patterns of cerebral blood flow reduction in patients with ischemic leukoaraiosis. $\mathrm{Neu}$ rology 2002;59:321-26

21. Zaidi SF, Aghaebrahim A, Urra X, et al. Final infarct volume is a stronger predictor of outcome than recanalization in patients with proximal middle cerebral artery occlusion treated with endovascular therapy. Stroke 2012;43:3238-44

22. Ay H, Arsava EM, Rosand J, et al. Severity of leukoaraiosis and susceptibility to infarct growth in acute stroke. Stroke 2008;39:1409-13

23. Rüber T, Schlaug G, Lindenberg R. Compensatory role of the cortico-rubro-spinal tract in motor recovery after stroke. Neurology 2012;79:515-22

24. Borich MR, Mang C, Boyd LA. Both projection and commissural pathways are disrupted in individuals with chronic stroke: investigating microstructural white matter correlates of motor recovery. BMC Neurosci 2012;13:107

25. Held V, Szabo K, Bazner H, et al. Chronic small vessel disease affects clinical outcome in patients with acute striatocapsular stroke. Cerebrovasc Dis 2012;33:86-91

26. Koton S, Schwammenthal Y, Merzeliak O, et al. Cerebral leukoaraiosis in patients with stroke or TIA: clinical correlates and 1-year outcome. Eur J Neurol 2009;16:218-25

27. Kang HJ, Stewart R, Park MS, et al. White matter hyperintensities and functional outcomes at 2 weeks and 1 year after stroke. Cerebrovasc Dis 2013;35:138-45

28. van Swieten JC, Hijdra A, Koudstaal PJ, et al. Grading white matter lesions on CT and MRI: a simple scale. J Neurol Neurosurg Psychiatry 1990;53:1080-83 